\section{GP186 AGE PROFILE OF CHILDREN AND ADOLESCENTS DIAGNOSED WITH INFLAMMATORY BOWEL DISEASE IN UNIVERSITY HOSPITAL LIMERICK OVER A 5 YEAR TIME PERIOD, 2014-2018}

Kerrie Hennigan*, Sarfarez Janjua, Michael Mahony. UHL, Limerick, Ireland

\subsection{6/archdischild-2019-epa.247}

Aim To investigate if there are any trends in the age at diagnosis of Inflammatory Bowel Disease (IBD) patients who attend the Paediatric Department at University Hospital Limerick.

Method Using our database of IBD patients, we analysed the charts of those diagnosed between Jan $1^{\text {st }} 2014$ and Dec $31^{\text {st }} 2018$. We extracted their date of birth, date of diagnosis, whether they were diagnosed with Ulcerative Colitis (UC) or Crohn's Disease (CD) and tabulated the results using Excel.

Results Over the time-period studied, in total 41 children and adolescents were diagnosed with IBD (59\% CD, 41\% UC). After stratifying by year there was no clear increase or decrease in the number of diagnoses per year from 2014 to 2018. The average age at diagnosis of IBD across this time period was 10.7 years (Range 2-16 years) and the average number of patients diagnosed per year was 8 (Range 5-11 patients per year).

When looking separately at those diagnosed with $\mathrm{CD}$, the average age of diagnosis across our 5-year time period was 10.48y (Range 2y-16y). When looking at those diagnosed with UC, the average age of diagnosis across our 5-year time period was $11.73 y$ (Range 4y-16y)

We analysed the age at diagnosis of both CD and UC by year in order to assess if there were any trends in age of diagnosis. For $\mathrm{CD}$, the average age of diagnoses are as follows: 2014; 10y (range $7 y$ to $12 y$ ), 2015; 11.4y (range 9y to $15 \mathrm{y}), 2016$; $13 \mathrm{y}$ (range $11 \mathrm{y}$ to $15 \mathrm{y}), 2017 ; 11.25 \mathrm{y}$ (4y to $16 y), 20186.75 y$ ( $2 y$ to $11 y)$. For UC, the average age of diagnoses are as follows; 2014; 10y (range 9y to 12y), 2015 $9.5 \mathrm{y}$ (4y to $13 \mathrm{y}), 2016 ; 12.67 \mathrm{y}$ (range $11 \mathrm{y}$ to $14 \mathrm{y}$ ), 2017; $14 \mathrm{y}$ (13y to $15 \mathrm{y}), 2019 ; 11.73 \mathrm{y}$ (8y to $15 \mathrm{y}$ ).

We then looked at trends in 'Very Early Onset IBD' (VEO IBD) patients, which are classified as those $<6$ years of age at diagnosis (1) and found that there has been a significant increase in those diagnosed with IBD in this age group during the period 2014-2018. In 2015 there was one VEO IBD diagnosis (UC). In 2017 there were two VEO IBD diagnoses and both were CD. Similarly, in 2018, there were two further VEO IBD diagnoses and again both were CD.

Discussion The average age at diagnosis remains relatively unchanged across the years with no obvious trend in either direction, however when analysing the subgroup of VEO IBD diagnoses, it is clear that for CD specifically there has been an increase in the number of children diagnosed under the age of 6 , with there being zero VEO IBD diagnoses from 2014-2016 and two per year in 2017 and 2018.

\section{REFERENCE}

1. Snapper SB. Very-Early-Onset Inflammatory Bowel Disease. Gastroenterol Hepatol (N Y). 2015;11(8):554-6.

\section{GP187 ANTHROPOMETRIC PARAMETERS IN CHILDREN WITH CELIAC DISEASE COMPLICATED BY IRON DEFICIENCY STATUS}

'Leonid Klimov, 'Marina Stoyan, 'Ekaterina Zavyalova, ${ }^{2}$ Irina Zakharova*,

${ }^{1}$ Victoria Kuryaninova. ${ }^{1}$ Stavropol State Medical University, Stavropol, Russian Federation;

${ }^{2}$ Russian Medical Continuing Postgraduate Academy, Moscow, Russian Federation

\subsection{6/archdischild-2019-epa.248}

Background and aims Analysis of the frequency of iron deficiency (ID) and the relationship with anthropometric parameters in children in the period of clinical manifestation of celiac disease (CD).

Methods 190 children with CD aged 8 months to 17 years $(4.6 \pm 0.3$ years), including $102(53.7 \%)$ girls and 88 $(46.3 \%)$ boys. Patients were divided into three groups depending on the iron supply. The first group 47 (24.7\%) patients without ID, the second group $87(45.8 \%)$ children with latent of ID, third group $56(29.5 \%)$ patients with ID anemia.The diagnosis was established in accordance with ESPGHAN criteria (1990, 2012). Indicators of physical development were evaluated by the program WHOAnthro and AnthroPlus.

Results The mean deviation of Z-score growth in the studied groups was $-0.8 \pm 0.2 \mathrm{SD},-1.1 \pm 0.1 \mathrm{SD}$ and $-1.8 \pm 0.2 \mathrm{SD}$. In children with IDA on the background of CD, growth retardation exceeds that in children without ID by 2.25 times $(\mathrm{p}<0.005)$, and in children with latent ID by 1.64 times $(p<0.005)$. Patients with IDA in $46.4 \%$ have growth retardation reaching the degree of somatogenic nanism, which is 2.38 times higher than in children with latent ID $(\mathrm{p}<0.001)$, and 1.98 times higher than in children without ID $(\mathrm{p}<0.05)$. The mean deviation of $\mathrm{Z}$-score body weight in the groups of patients was $-1.1 \pm 0.2 \mathrm{SD},-1.3 \pm 0.1 \mathrm{SD}$ and $-1.8 \pm 0.2$ SD. Body weight deficit in patients with IDA exceeds 1.64 times $(p<0.001)$ in children without ID and 1.38 times $(p<0.002)$ in children with latent ID. The correlation analysis showed a positive relationship between the deviation of Z-score growth and hemoglobin level $(r=0.24$, $\mathrm{p}<0.001)$, erythrocyte level $(\mathrm{r}=0.20, \mathrm{p}<0.01)$, serum ferritin level $(r=0.28, p<0.001)$, serum iron level $(r=0.17$, $\mathrm{p}<0.05)$; the inverse relationship with transferrin level $(\mathrm{r}=$ -0.28, $\mathrm{p}<0.001)$. There was also a positive correlation between Z-score body weight and hemoglobin level $(\mathrm{r}=0.24, \mathrm{p}<0.001)$, serum ferritin level $(\mathrm{r}=0.24, \mathrm{p}<0.001)$, serum iron level $(\mathrm{r}=0.21, \mathrm{p}<0.005)$; a negative correlation with transferrin level $(\mathrm{r}=-0.26, \mathrm{p}<0.001)$. A direct correlation between the presence of ID and stunting $(r=0.19$, $\mathrm{p}<0.01)$ and body mass deficit $(\mathrm{r}=0.14, \mathrm{p}<0.05)$ was demonstrated.

Conclusions Formation of delayed physical development in children with CD is accompanied by parallel development of latent ID and IDA due to impaired absorption, transport and progressive depletion of tissue iron stores. In children with CD complicated by ID, there is a more pronounced lag in the parameters of physical development than in patients without ID. 